\title{
Genome-wide association analysis identifies loci for left-sided displacement of the abomasum in German Holstein cattle
}

\author{
S. Mömke, ${ }^{\star}$ M. Sickinger,† P. Lichtner,‡ K. Doll, † J. Rehage, ,§ and O. Dist| ${ }^{\star 1}$ \\ *Institute for Animal Breeding and Genetics, University of Veterinary Medicine Hannover, Bünteweg 17p, 30559 Hannover, Germany \\ †Clinic for Ruminants and Swine, Faculty for Veterinary Medicine, Justus-Liebig-University Giessen, 35392 Giessen, Germany \\ łInstitute of Human Genetics, Helmholtz Zentrum München - German Research Center for Environmental Health, 85764 Neuherberg, Germany \\ $\S$ Clinic for Cattle, University of Veterinary Medicine Hannover, 30173 Hannover, Germany
}

\begin{abstract}
Left-sided displacement of the abomasum (LDA) is one of the most common disorders of the digestive system in many dairy breeds and particularly in Holstein dairy cows. We performed a genome-wide association study for 854 German Holstein cows, including 225 cases and 629 controls. All cows were genotyped using the Illumina Bovine SNP50 BeadChip (Illumina Inc., San Diego, CA). After quality control of genotypes, a total of 36,226 informative single nucleotide polymorphisms (SNP) were left for analysis. We used a mixed linear model approach for a genome-wide association study of LDA. In total, 36 SNP located on 17 bovine (Bos taurus) chromosomes (BTA) showed associations with LDA at nominal $-\log _{10} P$-values $>3.0$. Two of these SNP, located on BTA11 at $46.70 \mathrm{Mb}$ and BTA20 at 16.67 $\mathrm{Mb}$, showed genome-wide significant associations with LDA at $-\log _{10} P$-values $>4$.6. Pathway analyses indicated genes involved in calcium metabolism and insulin-dependent diabetes mellitus to be factors in the pathogenesis of LDA in German Holstein cows.
\end{abstract}

Key words: left-sided displacement of the abomasum, genome-wide association study, insulin, calcium

\section{INTRODUCTION}

German Holstein (GH) dairy cows dominate the milk production industry in Germany. Selection in this breed mainly focuses on milk performance, SCS, fertility, calving ease, and length of functional longevity to increase the economic profit. However, although selection for these desired traits has been accomplished through a total merit index, many frequent diseases in dairy cattle have not been considered yet. This is supposedly due to the difficulties of collecting individual disease data. Left-sided displacement of the abomasum

Received May 2, 2012.

Accepted February 12, 2013.

${ }^{1}$ Corresponding author: ottmar.distl@tiho-hannover.de
(LDA) is a common disease in high-performance dairy cows (Constable et al., 1992). In affected individuals, the abomasum bloats and rises dorsally to the left abdominal wall. This condition requires veterinary intervention and loss of productivity is often seen afterward, especially as LDA usually takes place around parturition or during early lactation. The main factors reported to precede LDA are a disturbed motility of the abomasum and an insufficient transport of gas (Van Winden and Kuiper, 2003). In GH, the prevalence for LDA was estimated at 1.2 to $2.6 \%$ (Wolf et al., 2001; Hamann et al., 2004) and can reach up to $7.5 \%$ in single herds (Poike and Fürll, 2000). The genetic component has a large effect on the incidence of LDA, as heritabilities have been estimated between 0.2 and 0.5 (Wolf et al., 2001; Hamann et al., 2004). Genome-wide association studies (GWAS) provide a step toward the identification of the underlying candidate genes for LDA and may open possibilities for selection against this disease. Genome-wide association studies are based upon SNP chip technology, which facilitates parallel genotyping of thousands of bovine SNP. In former GWAS, traits such as the susceptibility to Johne's disease (Settles et al., 2009), carcass traits (Kim et al., 2011), growth traits (Snelling et al., 2010), calving traits (Sahana et al., 2011), and fertility traits (Schulman et al., 2011) have been analyzed, among others. A genome-wide linkage analysis in GH half-sib families using highly polymorphic microsatellites identified 5 QTL for LDA on bovine (Bos taurus) chromosomes 1, 3, 21, 23, and 24 (Mömke et al., 2008). In the same study, further family-specific QTL were shown on BTA5, $6,10,12,15,16,17,19,23$, and 26 . The objective of the present study was to perform a GWAS using the Illumina Bovine SNP50 BeadChip (Illumina Inc., San Diego, CA) to identify SNP associated with LDA in GH cows and to use pathway analyses for networks of candidate genes. A set of LDA-associated candidate genes linked through common metabolic pathways might be a valuable tool for marker-assisted selection and for further research to understand the pathogenesis of LDA. 


\section{MATERIALS AND METHODS}

\section{Animals and Phenotypic Data}

We used GH cows for this study, as this dairy breed is most frequently affected by LDA in Germany. Ethylenediaminotetraacetate-blood samples were collected from more than 4,000 LDA-affected GH cows in 2006 to 2011. These animals were born between 1991 and 2009. Left-sided displacement of the abomasum was diagnosed at the Clinic for Cattle, University of Veterinary Medicine Hannover (Germany) or at the Clinic for Ruminants, Justus-Liebig-University (Giessen, Germany) and confirmed during LDA surgery. Samples of 4,200 LDA-unaffected GH control cows were collected in 2007 to 2009. These control cows were born in 1986 to 2008 and their phenotypes were secured by inspection of the individual veterinary reports. Out of all samples, a total of 854 cows were selected, including 225 LDA-affected and 629 nonaffected controls. These cows descended from 585 different sires and 549 different maternal grandsires.

\section{Genotyping}

Genomic DNA was extracted from the EDTA-blood samples using routine procedures. Genotyping was performed using the Illumina Bovine SNP50 BeadChip (Illumina Inc.), containing 54,001 SNP with an average spacing of $48.75 \mathrm{~kb}$. Raw data were analyzed using the genotyping module version 3.2.32 of the BeadStudio software (Illumina Inc.). All SNP underwent quality control (Purcell et al., 2007). Single nucleotide polymorphisms without a specified position $(\mathrm{n}=1,746)$, a minor allele frequency $<0.05(\mathrm{n}=15,328)$, a call rate $<90 \%$ ( $\mathrm{n}=473)$, or which significantly deviated from the Hardy-Weinberg equilibrium $(P<0.00001 ; \mathrm{n}=$ 228 ) were excluded from further analyses. Therefore, 36,226 SNP were left for the GWAS. The genotyping rate of the remaining SNP was $>0.98$ in the cows used for the analysis. All SNP positions were determined according to the Bos taurus UMD 3.1 assembly (Zimin et al., 2009).

\section{Statistical Analysis}

A mixed linear model (MLM) approach was used to identify SNP significantly associated with LDA. The model included the fixed genotype effects of the SNP and a random genetic effect of the animal through a SNP-based identity-by-state genomic relationship matrix and a heritability of 0.3 for LDA. The genomic relationship matrix was calculated using the formula given by VanRaden (2008) for computation of ge- nomic predictions. The different SNP genotypes were analyzed and, therefore, the genotypic effects included both additive and dominance effects. The genotype of each SNP was fitted as a fixed effect, one at a time, and the animal was treated as a random effect to parameterize quantitative genetic effects. The genomic relationship matrix among all individuals genotyped was derived from all 36,226 SNP used in the present GWAS. The analysis was performed using TASSEL software (version 3.0.88; Bradbury et al., 2007), which implements general linear model and MLM approaches for controlling population and family structure. We created quantile-quantile plots to show the observed versus expected $P$-value distribution across all SNP used in the GWAS. The observed $-\log _{10} P$-values were plotted against $-\log _{10} P$-values expected under the null hypothesis of independence. These plots obviously exhibited no evidence of overall systematic bias. Single nucleotide polymorphisms significantly associated with LDA show observed $-\log _{10} P$-values above the expected $-\log _{10} P$-values (Figure 1). Using a Bonferroni correction, an observed $-\log _{10} P$-value $>5.86$ corresponds to a significance level at $P<0.05$. However, a Bonferroni correction ignores correlations among SNP and, thus, leads to an overly conservative correction, particularly when marker density increases (Han et al., 2009). Odds ratios (OR) were determined based on the allele counts using the PROC CASECONTROL of SAS (version 9.3; 2011; SAS Institute Inc., Cary, NC).

\section{Pathway Analysis}

Functional pathway analyses were carried out using the Ingenuity Pathways Analysis (IPA; Ingenuity Systems, Redwood City, CA) software for 2 alternative sets of genes. The $P$-value in IPA is calculated using the right-tailed Fisher exact test. It considers the number of genes participating in a specific pathway and the total number of molecules known to be associated with that pathway in the Ingenuity knowledge base. The $P$-value, therefore, identifies statistically significant overrepresentation of the chosen genes in a pathway compared with the relation expected by chance (righttailed). For a first analysis, we chose all SNP at $-\log _{10}$ $P$-value $>3.0$. For each intragenic SNP, the specific gene was chosen (17 SNP). In the case of an intergenic SNP located between 2 genes, we chose both of these genes (12 SNP) and if only one gene was located within a distance of less than $500 \mathrm{~kb}$, only this gene was chosen (7 SNP). If no gene was located within a $500 \mathrm{~kb}$ distance of the SNP, no gene was included in the analysis (1 SNP). Therefore, a total of 46 different genes were chosen for the 37 SNP (Supplementary Table S1, available online at http://www.journalofdairyscience. 


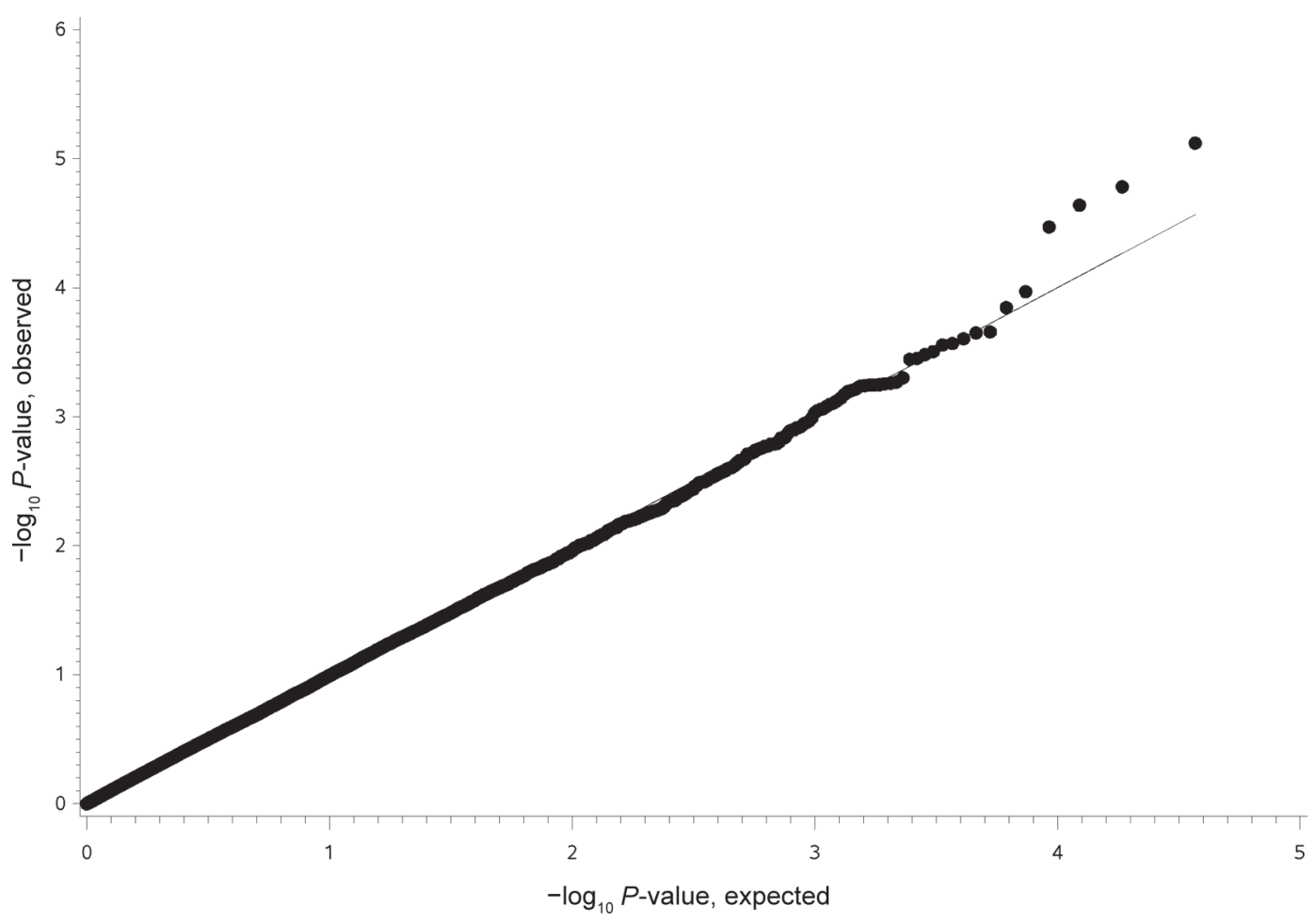

Figure 1. Observed versus expected $-\log _{10} P$-values of SNP significantly associated with left-sided displacement of the abomasum.

org/). Subsequently, we performed a second analysis, in which only the 16 genes that contained intragenic SNP (Supplementary Table S1) were used.

\section{RESULTS}

At a nominal significance level of a $-\log _{10} P$-value $>3.0$, we identified a total of $36 \mathrm{SNP}$ on 17 bovine chromosomes associated with LDA (Supplementary Table S2 and Supplementary Figure S1, available online at http://www.journalofdairyscience.org/). The phenotypic variance explained by a single SNP of the 36 LDA-associated SNP was 1.7 to $2.8 \%$ in the MLM analysis (Supplementary Table S3).

Three associated SNP, located on 2 different chromosomes showed significant associations with LDA at nominal $-\log _{10} P$-values $>4.0$ (Table 1 ). These were located on BTA11 $(46.70 \mathrm{Mb}, \mathrm{OR}=1.74$ and 102.89
$\mathrm{Mb}, \mathrm{OR}=1.53)$ and $20(16.67 \mathrm{Mb}, \mathrm{OR}=1.06)$. Two of these SNP were located within the genes IL1RN (BTA11) and $A K 8$ (BTA11). One SNP was intergenic, with IPO11 being the closest gene (BTA20). For these 3 SNP, both additive and dominance effects were significant in the MLM analysis and phenotypic variance explained by a single SNP reached values of 2.5 to $2.8 \%$ (Supplementary Table 3, available online at http:// www.journalofdairyscience.org/).

The distribution of genotypes among the unaffected and affected cows of the GWAS was evaluated for the 3 SNP at $-\log _{10} P$-values $>4.0$ (Table 2 ). The large effect of the SNP on BTA20 is caused by a very small number of LDA cows being homozygous for the minor allele and no unaffected cows homozygous for this genotype (Table 3). In our CASECONTROL analysis for this marker, $\chi^{2}$-values were significant only for the genotype distribution among cases and controls, but not for the

Table 1. Single nucleotide polymorphisms with their positions (UMD3.1 assembly; Zimin et al., 2009); identity; minor allele frequency for all $\left(\mathrm{MAF}\right.$ total), unaffected $\left(\mathrm{MAF}_{\mathrm{u}}\right)$, and affected $\left(\mathrm{MAF}_{\mathrm{a}}\right)$ cows; odds ratios $(\mathrm{OR})$ with $95 \% \mathrm{CI}$; and $-\log _{10} P$-values $(P)$ for all SNP associated with left-sided displacement of the abomasum (LDA) at nominal $-\log _{10} P$-value $>4.0$

\begin{tabular}{|c|c|c|c|c|c|c|c|c|c|}
\hline BTA position (bp) & SNP identity & $\begin{array}{l}\text { MAF } \\
\text { allele }\end{array}$ & $\begin{array}{l}\text { MAF } \\
\text { total }\end{array}$ & $\mathrm{MAF}_{\mathrm{u}}$ & $\mathrm{MAF}_{\mathrm{a}}$ & OR & Lower CI & Upper CI & $P$ \\
\hline 11-046701073 & ARS-BFGL-NGS-30536 & G & 0.12 & 0.10 & 0.17 & 1.74 & 1.74 & 1.28 & 4.64 \\
\hline $11-102885677$ & ARS-BFGL-NGS-28626 & G & 0.24 & 0.22 & 0.30 & 1.53 & 1.53 & 1.20 & 4.47 \\
\hline
\end{tabular}


Table 2. Distribution of the genotypes among left-sided displacement of the abomasum (LDA)-affected and unaffected cows for the 3 SNP with $-\log _{10} P$-values $>4.0^{1}$

\begin{tabular}{|c|c|c|c|c|c|c|c|c|}
\hline \multirow[b]{2}{*}{$\begin{array}{l}\text { BTA and position on } \\
\text { UMD3.1 }^{2} \text { assemby }^{2}(\mathrm{bp})\end{array}$} & \multirow[b]{2}{*}{ SNP identity } & \multirow{2}{*}{$\begin{array}{l}\text { MAF } \\
\text { allele }\end{array}$} & \multicolumn{6}{|c|}{ Genotype distribution among } \\
\hline & & & $\mathrm{AA}$ & $\mathrm{AB}$ & $\mathrm{BB}$ & $\mathrm{AA}$ & $\mathrm{AB}$ & $\mathrm{BB}$ \\
\hline $11-046701073$ & ARS-BFGL-NGS-30536 & $\mathrm{G}$ & 0.01 & 0.19 & 0.80 & 0.06 & 0.22 & 0.72 \\
\hline $11-102885677$ & ARS-BFGL-NGS-28626 & $\mathrm{G}$ & 0.03 & 0.38 & 0.59 & 0.11 & 0.38 & 0.51 \\
\hline $20-016672270$ & ARS-BFGL-NGS-114288 & $\mathrm{G}$ & 0.00 & 0.18 & 0.82 & 0.03 & 0.11 & 0.86 \\
\hline
\end{tabular}

${ }^{1}$ The minor allele (MAF allele) is indicated as $\mathrm{A}$ and the major allele as $\mathrm{B}$.

${ }^{2}$ Zimin et al. (2009).

allele distribution. For the SNP on BTA11, the highly LDA-susceptible homozygous genotypes were only represented by 15 and 45 cases, respectively.

Pathway analysis was at first carried out for 46 different genes corresponding to the SNP at nominal $-\log _{10} P$-values $>3.0$. Functional pathways significant at $-\log _{10} P$-values $>2.0$ were regarded. The complete data are provided in Supplementary Tables S4 and S5 (available online at http://www.journalofdairyscience. org/). A total of 56 different function annotations were detected for the set of genes. Of these, 8 contained clusters with more than 1 gene. The function annotations of these 8 pathways are quantity of corneocytes $\left(-\log _{10}\right.$ $P$-value $=5.35 ; 2$ genes $)$, deposition of $\mathrm{Ca}^{2+}\left(-\log _{10}\right.$ $P$-value $=3.28 ; 2$ genes $),$ morphology of skin $\left(-\log _{10}\right.$ $P$-value $=2.5 ; 3$ genes $)$, insulin-dependent diabetes mellitus $\left(-\log _{10} P\right.$-value $=2.34 ; 4$ genes $)$, quantity of blood vessels $\left(-\log _{10} P\right.$-value $=2.32 ; 2$ genes $)$, fear $(-$ $\log _{10} P$-value $=2.18 ; 2$ genes $)$, burn $\left(-\log _{10} P\right.$-value $=$ $2.16 ; 2$ genes $)$, and binding of $\mathrm{T}$ lymphocytes $\left(-\log _{10}\right.$ $P$-value $=2.0 ; 2$ genes $)$. The most probable pathways for the pathogenesis of LDA are those of deposition of $\mathrm{Ca}^{2+}$, containing the genes BMP2K and SLC8A1, and the insulin-dependent diabetes mellitus, containing the genes CUX2, IL1RN, LRP1B, and POLR2M. In a second pathway analysis, we used only those genes that contained the 16 intragenic SNP. In this analysis, a total of 14 different function annotations contained clusters with more than 1 gene. The most probable function annotation was deposition of $\mathrm{Ca}^{2+}\left(-\log _{10} P\right.$ - value $=4.17)$. Two further functions detected in this second analysis were also found in the first one. These functions are burn $\left(-\log _{10} P\right.$-value $\left.=3.04\right)$ and insulindependent diabetes mellitus $\left(-\log _{10} P\right.$-value $\left.=1.63\right)$.

\section{DISCUSSION}

Correction for multiple testing was performed using the method suggested by Lander and Kruglyak (1995) for calculation the threshold of genome-wide significance as used in the GWAS of Schulman et al. (2011). The threshold for significance was at a $-\log _{10} P$-value of 4.6 in this latter study. Using this threshold in the present study for LDA, the SNP on BTA11 (46.7 Mb) and $20(16.7 \mathrm{Mb})$ are genome-wide significant. However, the SNP on BTA11 (102.9 Mb) is just below this threshold. Using strictly conservative methods for the significance threshold, such as the Bonferroni correction for all SNP used in the present GWAS, there are no genome-wide significant results.

The SNP on BTA20 (16.7 Mb) shows significant association with LDA only for the genotypes, but not for alleles. This is reflected by a low OR for this SNP. Although the association of this marker, therefore, might be an artifact, it also might contribute information to the genetics of LDA, especially by means of the rare allele.

Of the SNP at $-\log _{10} P$-values $>3.0$, some are located within or nearby the previously identified QTL using a whole-genome scan for LDA in a linkage study

Table 3. Genotypic distribution for the $3 \mathrm{SNP}$ at $-\log _{10} P$-values $>4.0$ in the total number of cows and the proportions of the genotypes in left-sided displacement of the abomasum (LDA)-affected cows ${ }^{1}$

\begin{tabular}{|c|c|c|c|c|c|c|c|}
\hline \multirow{2}{*}{$\begin{array}{l}\text { BTA position on } \\
\text { UMD3.1 }^{2} \text { assembly } \\
\text { (bp) }\end{array}$} & \multirow[b]{2}{*}{ SNP identity } & \multicolumn{3}{|c|}{ Number of cows } & \multicolumn{3}{|c|}{$\begin{array}{c}\text { Proportion of } \\
\text { LDA-affected cows }\end{array}$} \\
\hline & & AA & $\mathrm{AB}$ & BB & AA & $\mathrm{AB}$ & BB \\
\hline $11-102885677$ & ARS-BFGL-NGS-28626 & 45 & 319 & 475 & 0.56 & 0.27 & 0.24 \\
\hline 20-016672270 & ARS-BFGL-NGS-114288 & 7 & 137 & 707 & 1.00 & 0.18 & 0.27 \\
\hline
\end{tabular}

${ }^{1}$ The minor allele (MAF allele) is indicated as A and the major allele as $\mathrm{B}$.

${ }^{2}$ Zimin et al. (2009). 
(Supplementary Table S6, available online at http:// www.journalofdairyscience.org/; Mömke et al., 2008). The LDA-associated SNP at $57.91\left(-\log _{10} P\right.$-value $=$ 3.2) on BTA24 from the GWAS is located within a QTL of the previous linkage analysis. Furthermore, an LDA-associated SNP $\left(-\log _{10} P\right.$-value $\left.>3.0\right)$ on BTA19 is located within a family-specific QTL and a SNP on BTA17 is located $2.9 \mathrm{Mb}$ distant of a family-specific QTL from the linkage study. A possible reason for differences among the present GWAS and the previous linkage study for LDA may be due to the half-sib structure in the linkage analysis. The LDA-linked regions were dependent on 14 different paternal half-sib families and their segregation pattern for LDA-specific QTL. On the other hand, chromosomal regions may not be associated with LDA if linkage is caused by individual sire families and the underlying mutations are restricted to these few sire families. In the present GWAS, LDA-associated regions were detected on chromosomal positions different from the linkage study due to the diverse ancestry of the animals included in the analysis.

The abomasum in ruminants is the equivalent of the stomach in monogastric species. Besides cattle and humans, gastric dilatation and displacement has also been reported in other species such as dogs (bloat; Monnet, 2003) and pigs (Bethlehem and Hilvering, 1987). However, causal genes have not been identified in these species so far. Previous studies on the pathogenetic changes related to LDA were carried out in vitro or examining cows already affected by LDA. These studies suggested a preceding decreased motility of the abomasum and malfunctions at the level of the intrinsic nervous system combined with impaired cholinergic muscle responses. Cows developing LDA in most instances showed impaired abomasal emptying and abomasal atony (Geishauser et al., 1998). Disturbed motility of the abomasum and insufficient transport of gas usually precede LDA (Van Winden and Kuiper, 2003). Causes discussed to be responsible for decreased motility and abomasal atony in cows with LDA are insulin concentration, the effect of the vagus nerve, blood calcium level, potassium homeostasis, and metabolic alkalosis (Van Winden and Kuiper, 2003; Pravettoni et al., 2004; Türck and Leonhard-Marek, 2010). Therefore, candidate genes for LDA might affect insulin metabolism, calcium homeostasis, nerves, neurotransmitters, hormones or their receptors, and other factors that influence gastric motility or digestion.

Of the $3 \mathrm{SNP}$ associated with LDA at $-\log _{10} P$-values $>4.0$, those on BTA11 (46.70 and $102.89 \mathrm{Mb}$ ) are located within the genes $I L 1 R N$ and $A K 8$. Therefore, these genes can be regarded as positional candidate genes. Whereas the few facts currently known about the function of $A K 8$ show no obvious connection with LDA, only $I L 1 R N$ can be regarded as a positional as well as functional candidate gene for LDA. In human pancreatic $\beta$-cells of patients with type 2 diabetes, decreased expression of $I L 1 R N$ was observed (Maedler et al., 2004). In vitro, chronic exposure of human islets to leptin, which was shown to be associated with LDA before (Chebel et al., 2008), decreased $\beta$-cell production of $I L 1 R N$, leading to impaired $\beta$-cell function and apoptosis. Supplementation of $I L 1 R N$ protected human islets from the destructive effect of leptin. The gene also provides protection against glucose-induced production of IL-1 $\beta$ in human pancreatic $\beta$-cells. This affects insulin secretion, cell proliferation, and apoptosis.

In the pathway analysis, IL1RN combined with the $C U X 2, L R P 1 B$, and POLR2M gene cluster for the function annotation insulin-dependent diabetes mellitus. Of these 4 genes, IL1RN and CUX2 contain intragenic LDA-associated SNP. The diabetes mellitus pathway contains the biggest number of genes of all pathways resulting from the analysis. Diabetes mellitus is a metabolic disorder, caused by a deficiency of insulin, causing hyperglycemia. Hyperglycemia is often found in LDA-affected cows (Van Winden and Kuiper, 2003) and was shown to cause a reduced outflow of abomasal fluid off the abomasum in dairy cows and an elevated plasma glucose level; thus, it was considered a potential risk factor in the pathogenesis of LDA (Holtenius et al., 2000). Another candidate functional pathway for LDA showing even higher probabilities was calcium metabolism. It comprises the genes BMP2K and SLC8A1, both containing intragenic LDA-associated SNP. An impaired calcium homeostasis was already shown to be related to LDA (Van Winden and Kuiper, 2003). Although function annotations, such as quantity of blood vessels or fear, might have an effect on abomasal motility, they are not likely to be predominantly involved in LDA pathogenesis. The remaining pathways do show no obvious relationships with LDA either.

Much research has been done before to demonstrate correlations between metabolic alterations, myoelectrical activities of the abomasum, and LDA. However, those analyses had to be conducted in vitro or on cows already suffering from LDA. In the present study, we searched for a framework for the pathogenesis of LDA using a pathway analysis for positional candidate genes ascertained through GWAS. This analysis gave hints that calcium metabolism as well as insulin metabolism may be important pathogenic factors for LDA in $\mathrm{GH}$ cows. These insights into the possible role of networks of genes causing susceptibility to LDA may provide a primary framework for the pathogenesis of LDA. 


\section{CONCLUSIONS}

In this study, we identified 36 LDA-associated SNP on 17 chromosomes and analyzed the pathways of the positional candidate genes for these SNP. Two SNP were associated with LDA at $-\log _{10} P$-values $>4$.6. Candidate genes involved in pathways of calcium metabolism and insulin-dependent diabetes mellitus may be players in the genetic pathogenesis of LDA in GH cows.

\section{ACKNOWLEDGMENTS}

This study and S. Mömke were supported by grants of the German Research Council (DFG, Bonn, Germany; MO1691/2-1).

\section{REFERENCES}

Bethlehem, M., and J. Hilvering. 1987. Stomach dilatation-stomach torsion syndrome: A 'new' clinical picture in sows? Tijdschr. Diergeneeskd. 112:1101-1105. (In Dutch).

Bradbury, P. J., Z. Zhang, D. E. Kroon, T. M. Casstevens, Y. Ramdoss, and E. S. Buckler. 2007. TASSEL: Software for association mapping of complex traits in diverse samples. Bioinformatics 23:2633-2635.

Chebel, R. C., F. Susca, and J. E. P. Santos. 2008. Leptin genotype is associated with lactation performance and health of Holstein cows. J. Dairy Sci. 91:2893-2900.

Constable, P. D., G. Y. Miller, G. F. Hoffsis, B. L. Hull, and D. M. Rings. 1992. Risk factors for abomasal volvulus and left abomasal displacement in cattle. Am. J. Vet. Res. 53:1184-1192.

Geishauser, T., D. Reiche, and M. Schemann. 1998. In vitro motility disorders associated with left-sided displaced abomasum in dairy cows. Neurogastroenterol. Motil. 10:395-401.

Hamann, H., V. Wolf, H. Scholz, and O. Distl. 2004. Relationships between lactational incidence of displaced abomasum and milk production traits in German Holstein cows. J. Vet. Med. A Physiol. Pathol. Clin. Med. 51:203-208.

Han, B., H. M. Kang, and E. Eskin. 2009. Rapid and accurate multiple testing correction and power estimation for millions of correlated markers. PLoS Genet. 5:e1000456.

Holtenius, K., K. Sternbauer, and P. Holtenius. 2000. The effect of the plasma glucose level on the abomasal function in dairy cows. J. Anim. Sci. 78:1930-1935

Kim, Y., J. Ryu, J. Woo, J. B. Kim, C. Y. Kim, and C. Lee. 2011. Genome-wide association study reveals five nucleotide sequence variants for carcass traits in beef cattle. Anim. Genet. 42:361-365.

Lander, E., and L. Kruglyak. 1995. Genetic dissection of complex traits: Guidelines for interpreting and reporting linkage results. Nat. Genet. 11:241-247.

Maedler, K., P. Sergeev, J. A. Ehses, Z. Mathe, D. Bosco, T. Berney, J.-M. Dayer, M. Reinecke, P. A. Halban, and M. Y. Donath. 2004
Leptin modulates $\beta$ cell expression of IL-1 receptor antagonist and release of IL-1 $\beta$ in human islets. Proc. Natl. Acad. Sci. USA 101:8138-8143.

Mömke, S., H. Scholz, K. Doll, J. Rehage, and O. Distl. 2008. Mapping quantitative trait loci for left-sided displaced abomasum in German Holstein dairy cows. J. Dairy Sci. 91:4383-4392.

Monnet, E. 2003. Gastric dilatation-volvulus syndrome in dogs. Vet. Clin. North Am. Small Anim. Pract. 33:987-1005.

Poike, A., and M. Fürll. 2000. Zur Epidemiologie der Labmagenverlagerung (dislocatio abomasi) in Mitteldeutschland. Pages 29-39 in Ätiologie, Pathogenese, Diagnostik, Prognose, Therapie und Prophylaxe der Dislocatio abomasi. Proc. Intl. Workshop Leipzig 1998. M. Fürll, ed. Universitätsverlag Leipzig, Germany.

Pravettoni, D., K. Doll, M. Hummel, E. Cavallone, M. Re, and A. G. Belloli. 2004. Insulin resistance and abomasal motility disorders in cows detected by use of abomasoduodenal electromyography after surgical correction of left left-sided displaced abomasum. Am. J. Vet. Res. 65:1319-1324.

Purcell, S., B. Neale, K. Todd-Brown, L. Thomas, M. A. R. Ferreira, D. Bender, J. Maller, P. Sklar, P. I. W. de Bakker, M. J. Daly, and P. C. Sham. 2007. PLINK: A toolset for whole-genome association and population-based linkage analysis. Am. J. Hum. Genet. $81: 559-575$

Sahana, G., B. Guldbrandtsen, and M. S. Lund. 2011. Genome-wide association study for calving traits in Danish and Swedish Holstein cattle. J. Dairy Sci. 94:479-486.

Schulman, N. F., G. Sahana, T. Iso-Touru, S. D. McKay, R. D. Schnabel, M. S. Lund, J. F. Taylor, J. Virta, and J. H. Vilkki. 2011. Mapping of fertility traits in Finnish Ayrshire by genome-wide association analysis. Anim. Genet. 42:263-269.

Settles, M., R. Zanella, S. D. McKay, R. D. Schnabel, J. F. Taylor, R. Whitlock, Y. Schukken, J. S. Van Kessel, J. M. Smith, and H. Neibergs. 2009. A whole genome association analysis identifies loci associated with Mycobacterium avium ssp. paratuberculosis infection status in US Holstein cattle. Anim. Genet. 40:655-662.

Snelling, W. M., M. F. Allan, J. W. Keele, L. A. Kuehn, T. McDaneld, T. P. L. Smith, T. S. Sonstegard, R. M. Thallman, and G. L. Bennett. 2010. Genome-wide association study of growth in crossbred beef cattle. J. Anim. Sci. 88:837-848.

Türck, G., and S. Leonhard-Marek. 2010. Potassium and insulin affect the contractility of abomasal smooth muscle. J. Dairy Sci. 93:3561-3568.

Van Winden, S. C. L., and R. Kuiper. 2003. Left displacement of the abomasum in dairy cattle: Recent developments in epidemiological and etiological aspects. Vet. Res. 34:47-56.

VanRaden, P. M. 2008. Efficient methods to compute genomic predictions. J. Dairy Sci. 91:4414-4423.

Wolf, V., H. Hamann, H. Scholz, and O. Distl. 2001. Influences on the occurrence of abomasal displacements in German Holstein cows. Dtsch. Tierärztl. Wochenschr. 108:403-408. (In German).

Zimin, A. V., A. L. Delcher, L. Florea, D. R. Kelley, M. C. Schatz, D. Puiu, F. Hanrahan, G. Pertea, C. P. Van Tassell, T. S. Sonstegard, G. Marçais, M. Roberts, P. Subramanian, J. A. Yorke, and S. L. Salzberg. 2009. A whole-genome assembly of the domestic cow, Bos taurus. Genome Biol. 10:R42. 